\title{
Combinatorial formulas for some generalized Ekeland-Hofer-Zehnder capacities of convex polytopes
}

\author{
Kun Shi and Guangcun Lu* \\ September 13, 2021 \\ Dedicated to Professor Claude Viterbo on the occasion of his sixtieth birthday
}

\begin{abstract}
Motivated by Pazit Haim-Kislev's combinatorial formula for the Ekeland-Hofer-Zehnder capacities of convex polytopes, we give corresponding formulas for $\Psi$-Ekeland-Hofer-Zehnder and coisotropic Ekeland-Hofer-Zehnder capacities of convex polytopes introduced by the second named author and others recently. Contrary to Pazit Haim-Kislev's subadditivity result for the Ekeland-Hofer-Zehnder capacities of convex domains, we show that the coisotropic HoferZehnder capacities satisfy the superadditivity for suitable hyperplane cuts of two-dimensional convex domains.
\end{abstract}

\section{Contents}

1 Introduction and results 1

2 Preliminaries 5

3 Piecewise affine paths

4 Proof of Theorem $1.1 \quad 10$

5 Proofs of Theorems 1.2, 1.3

\section{Introduction and results}

Symplectic capacities are important invariants in studies of symplectic topology. Different symplectic capacities measure the "symplectic size" of sets from different views. Precise computations of them are usually difficult.

For a compact convex domain $K$ with smooth boundary $\mathcal{S}=\partial K$ in the standard symplectic Euclidean space $\left(\mathbb{R}^{2 n}, \omega_{0}\right)$, Ekeland-Hofer [6] (see also [16]) and Hofer-Zehnder [8] showed,

\footnotetext{
${ }^{*}$ Corresponding author

Partially supported by the NNSF 11271044 of China.

2010 Mathematics Subject Classification. 53D05, 52B12 (primary), 53D05, 53C23 (secondary).
} 
respectively, that its Ekeland-Hofer capacity $c_{\mathrm{EH}}(K)$ and Hofer-Zehnder capacity $c_{\mathrm{HZ}}(K)$ were equal to

$$
c_{\mathrm{EHZ}}(K):=\min \{A(x)>0 \mid x \text { is a closed characteristic on } \mathcal{S}\}
$$

(called the Ekeland-Hofer-Zehnder capacity below), where by a closed characteristic on $\mathcal{S}$ we mean a $C^{1}$ embedding $z$ from $S^{1}=[0, T] /\{0, T\}$ into $\mathcal{S}$ satisfying $\dot{z}(t) \in\left(\mathcal{L}_{\mathcal{S}}\right)_{z(t)}$ for all $t \in[0, T]$, where

$$
\mathcal{L}_{\mathcal{S}}=\left\{(x, \xi) \in T \mathcal{S} \mid \omega_{0 x}(\xi, \eta)=0, \forall \eta \in T_{x} \mathcal{S}\right\}
$$

and the action of a path $z \in W^{1,2}\left([0, T], \mathbb{R}^{2 n}\right)$ is defined by

$$
A(z)=\frac{1}{2} \int_{0}^{T}\langle-J \dot{z}, z\rangle d t
$$

with $J=\left(\begin{array}{cc}0 & -I_{n} \\ I_{n} & 0\end{array}\right)$, where $z \in W^{1,2}\left([0, T], \mathbb{R}^{2 n}\right)$ if $z$ is absolutely continuous and

$$
\int_{0}^{T}\|z(t)\|^{2} d t<\infty \text { and } \int_{0}^{T}\|\dot{z}(t)\|^{2} d t<\infty .
$$

We equip $H^{1}\left([0, T], \mathbb{R}^{2 n}\right):=W^{1,2}\left([0, T], \mathbb{R}^{2 n}\right)$ the natural Sobolev norm:

$$
\|z\|_{W^{1,2}}:=\left(\int_{0}^{T}\|z(t)\|^{2}+\|\dot{z}(t)\|^{2} d t\right)^{\frac{1}{2}} .
$$

When the smoothness assumption of the boundary $\mathcal{S}$ is thrown away, then (1.1) is still true if "closed characteristic" in the right side of (1.1) may be replaced by "generalized closed characteristic", where a generalized closed characteristic on $\mathcal{S}$ is a $T$-periodic nonconstant absolutely continuous curve $z: \mathbb{R} \rightarrow \mathbb{R}^{2 n}$ (for some $T>0$ ) such that $z(\mathbb{R}) \subset \mathcal{S}$ and $\dot{z}(t) \in J N_{\mathcal{S}}(z(t)$ ) a.e., where $N_{\mathcal{S}}(x)=\left\{y \in \mathbb{R}^{2 n} \mid\langle u-x, y\rangle \leqslant 0, \forall u \in K\right\}$ is the normal cone to $K$ at $x \in \mathcal{S}$. The action of such a generalized closed characteristic $x:[0, T] \rightarrow \mathcal{S}$ is still defined by (1.2).

In general, it is difficult to compute $c_{\mathrm{EHZ}}(K)$ by finding minimal closed characteristics with (1.1). If $K$ is a convex polytope with $(2 n-1)$-dimensional facets $\left\{F_{i}\right\}_{i=1}^{\mathbf{F}_{K}}, n_{i}$ is the unit outer normal to $F_{i}$, and $h_{i}=h_{K}\left(n_{i}\right)$ the "oriented height" of $F_{i}$ given by the support function of $K, h_{K}(y):=\sup _{x \in K}\langle x, y\rangle$, starting from (1.1) Pazit Haim-Kislev [15] recently established the following beautiful combinatorial formula for $c_{\mathrm{EHZ}}(K)$ :

$$
c_{\mathrm{EHZ}}(K)=\frac{1}{2}\left[\max _{\sigma \in S_{\mathbf{F}_{K}},\left(\beta_{i}\right) \in M(K)} \sum_{1 \leq j<i \leq \mathbf{F}_{K}} \beta_{\sigma(i)} \beta_{\sigma(j)} \omega_{0}\left(n_{\sigma(i)}, n_{\sigma(j)}\right)\right]^{-1},
$$

where $S_{\mathbf{F}_{K}}$ is the symmetric group on $\mathbf{F}_{K}$ letters and

$$
M(K)=\left\{\left(\beta_{i}\right)_{i=1}^{\mathbf{F}_{K}} \mid \beta_{i} \geq 0, \sum_{i=1}^{\mathbf{F}_{K}} \beta_{i} h_{i}=1, \sum_{i=1}^{\mathbf{F}_{K}} \beta_{i} n_{i}=0\right\} .
$$

As an important application, Pazit Haim-Kislev [15] proved a subadditivity property of the capacity $c_{\mathrm{EHZ}}$ for hyperplane cuts of arbitrary convex domains, which solved a special case of the subadditivity conjecture for capacities ([2]).

Recently, motivated by Clarke [3, 4] and Ekeland [7] Rongrong Jin and the second named author introduced relative versions (or generalizations) of the Ekeland-Hofer capacity and the Hofer-Zehnder capacity in [10]. Precisely, for a symplectic manifold $(M, \omega)$ and for a 
$\Psi \in \operatorname{Symp}(M, \omega)$ with $\operatorname{Fix}(\Psi) \neq \emptyset$, we defined a relative version of the Hofer-Zehnder capacity $c_{\mathrm{HZ}}(M, \omega)$ of $(M, \omega)$ with respect to $\Psi, c_{\mathrm{HZ}}^{\Psi}(M, \omega)$, which becomes $c_{\mathrm{HZ}}(M, \omega)$ if $\Psi=i d_{M}$. For a symplectic matrix $\Psi \in \operatorname{Sp}(2 n, \mathbb{R})$ with $\operatorname{Fix}(\Psi) \neq \emptyset$, and for each $B \subset \mathbb{R}^{2 n}$ such that $B \cap \operatorname{Fix}(\Psi) \neq \emptyset$, we also introduced a relative version of the Ekeland-Hofer capacity $c_{\mathrm{EH}}(B)$ of $B$ with respect to $\Psi, c_{\mathrm{EH}}^{\Psi}(B)$, which becomes $c_{\mathrm{EH}}(B)$ if $\Psi=I_{2 n}$. If a compact convex domain $K \subset \mathbb{R}^{2 n}$ with boundary $\mathcal{S}=\partial K$ contains a fixed point of $\Psi \in \operatorname{Sp}(2 n, \mathbb{R})$ in the interior of it, we proved in [10:

$$
c_{\mathrm{EH}}^{\Psi}(K)=c_{\mathrm{HZ}}^{\Psi}(K)=\min \{A(x)>0 \mid x \text { is a generalized } \Psi \text {-characteristic on } \mathcal{S}\},
$$

where a generalized $\Psi$-characteristic on $\mathcal{S}$ is a nonconstant absolutely continuous curve $z$ : $[0, T] \rightarrow \mathbb{R}^{2 n}$ (for some $T>0$ ) such that $z([0, T]) \subset \mathcal{S}, z(T)=\Psi z(0)$ and $\dot{z}(t) \in J N_{\mathcal{S}}(z(t)$ ) a.e., where $N_{\mathcal{S}}(x)$ is the normal cone to $K$ at $x \in \mathcal{S}$ as above, and the action $A(z)$ of $z$ is still defined by (1.2). (If $\mathcal{S}$ is $C^{1,1}$-smooth, "generalized closed characteristic" in the right side of (1.4) may be replaced by "closed characteristic", where a $\Psi$-characteristic on $\mathcal{S}$ is a $C^{1}$ embedding $z$ from $[0, T]$ (for some $T>0$ ) into $\mathcal{S}$ such that $z(T)=\Psi z(0)$ and $\dot{z} \in\left(\mathcal{L}_{\mathcal{S}}\right)_{z(t)}$ for all $t \in[0, T]$ ). Our first result is an analogue of (1.3) for $c_{\mathrm{EHZ}}^{\Psi}(K):=c_{\mathrm{EH}}^{\Psi}(K)=c_{\mathrm{HZ}}^{\Psi}(K)$.

Theorem 1.1. Let $K$ be a convex polytope as above (1.3). Suppose that $\Psi \in \operatorname{Sp}(2 n, \mathbb{R})$ has a fixed point sitting in the interior of $K$. Then

$$
c_{\mathrm{EHZ}}^{\Psi}(K)=\min _{\left(\left(\beta_{i}\right)_{i=1}^{\left.\mathbf{F}_{K}, v, \sigma\right)} \in M_{\Psi}(K)\right.} \frac{2}{4 \sum_{1 \leqslant j<i \leqslant \mathbf{F}_{K}} \beta_{\sigma(i)} \beta_{\sigma(j)} \omega_{0}\left(n_{\sigma(j)}, n_{\sigma(i)}\right)-\omega_{0}(\Psi v, v)},
$$

where

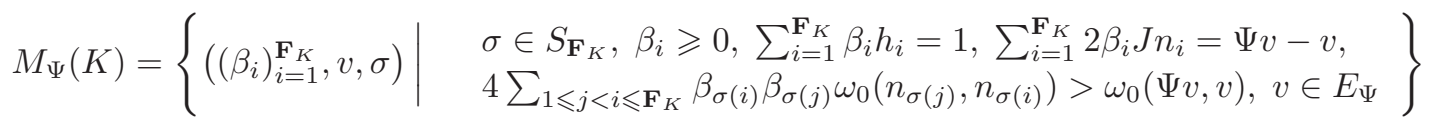
with $E_{\Psi}$ being the orthogonal complement of $\operatorname{Ker}\left(\Psi-I_{2 n}\right)$ in $\mathbb{R}^{2 n}$.

Note: Under our convention $\langle x, y\rangle=\omega_{0}(x, J y), \omega_{0}\left(n_{\sigma(i)}, n_{\sigma(j)}\right)$ in (1.3) should be changed into $\omega_{0}\left(n_{\sigma(j)}, n_{\sigma(i)}\right)$.

Lisi and Rieser [13] introduced the notion of a coisotropic capacity and constructed a coisotropic Hofer-Zehnder capacity, which is a relative version of the Hofer-Zehnder capacity with respect to a coisotropic submanifold. Rongrong Jin and the second named author recently constructed a relative version of the Ekeland-Hofer capacity with respect to a special class of coisotropic subspaces in [12]. Consider coisotropic subspaces of $\left(\mathbb{R}^{2 n}, \omega_{0}\right)$,

$$
\mathbb{R}^{n, k}=\left\{x \in \mathbb{R}^{2 n} \mid x=\left(q_{1}, \cdots, q_{n}, p_{1}, \cdots, p_{k}, 0, \cdots, 0\right)\right\}, \quad k=0, \cdots, n .
$$

The isotropic leaf through $x \in \mathbb{R}^{n, k}$ is $x+V_{0}^{n, k}$, where

$$
V_{0}^{n, k}=\left\{x \in \mathbb{R}^{2 n} \mid x=\left(0, \cdots, 0, q_{k+1}, \cdots, q_{n}, 0, \cdots, 0\right)\right\} .
$$

The leaf relation $\sim$ on $\mathbb{R}^{n, k}$ is that $x \sim y$ if and only if $y \in x+V_{0}^{n, k}$. From now on we fix an integer $0 \leqslant k<n$ and assume that $K \subset \mathbb{R}^{2 n}$ is a compact convex domain with $C^{1,1}$-smooth boundary $\mathcal{S}=\partial K$ and satisfying $\operatorname{Int}(K) \cap \mathbb{R}^{n, k} \neq \emptyset$. A nonconstant absolutely continuous curve $z:[0, T] \rightarrow \mathbb{R}^{2 n}$ (for some $T>0$ ) is called a generalized leafwise chord (abbreviated GLC) on $\mathcal{S}$ for $\mathbb{R}^{n, k}$ if $z([0, T]) \subset \mathcal{S}, \dot{z}(t) \in J N_{\mathcal{S}}(z(t))$ a.e., $z(0), z(T) \in \mathbb{R}^{n, k}$ and $z(0)-z(T) \in V_{0}^{n, k}$. The action $A(z)$ of such a chord is still defined by (1.2). In [11, 12] Rongrong Jin and the second named author proved respectively that the coisotropic HoferZehnder capacity $c_{\mathrm{LR}}\left(K, K \cap \mathbb{R}^{n, k}\right)$ of $K$ relative to $\mathbb{R}^{n, k}$ and the coisotropic Ekeland-Hofer capacity $c^{n, k}(K)$ of $K$ relative to $\mathbb{R}^{n, k}$ satisfy

$$
c_{\mathrm{LR}}\left(K, K \cap \mathbb{R}^{n, k}\right)=c^{n, k}(K)=\min \left\{A(x)>0 \mid x \text { is a GLC on } \mathcal{S} \text { for } \mathbb{R}^{n, k}\right\} .
$$


Here is our second result.

Theorem 1.2. Let $K$ be a convex polytope as above (1.3). Suppose $K \cap \mathbb{R}^{n, k} \neq \emptyset$. Then

$$
c_{\mathrm{LR}}\left(K, K \cap \mathbb{R}^{n, k}\right)=\frac{1}{2} \min _{\left(\left(\beta_{i}\right)_{i=1}^{\left.\mathbf{F}_{K}, \sigma\right) \in M(K)}\right.} \frac{1}{\sum_{1 \leqslant j<i \leqslant \mathbf{F}_{K}} \beta_{\sigma(i)} \beta_{\sigma(j)} \omega_{0}\left(n_{\sigma(j)}, n_{\sigma(i)}\right)},
$$

where

$$
M(K)=\left\{\left(\left(\beta_{i}\right)_{i=1}^{\mathbf{F}_{K}}, \sigma\right) \mid \begin{array}{l}
\beta_{i} \geqslant 0, \sum_{i=1}^{\mathbf{F}_{K}} \beta_{i} h_{i}=1, \sum_{i=1}^{\mathbf{F}_{K}} \beta_{i} J n_{i} \in V_{0}^{n, k}, \\
\quad \sum_{1 \leqslant j<i \leqslant \mathbf{F}_{K}} \beta_{\sigma(i)} \beta_{\sigma(j)} \omega_{0}\left(n_{\sigma(j)}, n_{\sigma(i)}\right)>0, \sigma \in S_{\mathbf{F}_{K}}
\end{array}\right\} .
$$

Unlike Ekeland-Hofer-Zehnder capacity, one cannot expect that the coisotropic HoferZehnder capacity satisfies the subadditivity as stated in [15. Theorem 1.8] in general. In fact, when $n=1$ and $k=0$, our following result is opposite to the expected one.

Theorem 1.3. Let $D \subset \mathbb{R}^{2}$ be a convex domain satisfying $D \cap \mathbb{R}^{1,0} \neq \emptyset$, and let $L \subset \mathbb{R}^{2}$ be a straight line through $D$ such that $L \neq \mathbb{R}^{1,0}$ and $D \cap L \cap \mathbb{R}^{1,0} \neq \emptyset$. Denote by $D_{1}$ and $D_{2}$ the two parts divided by $L$. Then

$$
c_{\mathrm{LR}}\left(D, D \cap \mathbb{R}^{1,0}\right) \geq c_{\mathrm{LR}}\left(D_{1}, D_{1} \cap \mathbb{R}^{1,0}\right)+c_{\mathrm{LR}}\left(D_{2}, D_{2} \cap \mathbb{R}^{1,0}\right) .
$$

Remark 1.4. Inequality (1.7) is sharp, and it can be strict in some cases. Consider the following example. Let $P=\{(x, y)|| x|\leqslant 1| y \mid, \leqslant 1\}$ and $L=\{(x, x) \mid x \in \mathbb{R}\}$. Then $L$ divides $P$ into two parts $P_{1}:=\{(x, y) \mid x \leqslant y\} \cap P$ and $P_{2}:=\{(x, y) \mid x \geqslant y\} \cap P$. Using Theorem 1.2 we can easily compute $c_{\mathrm{LR}}\left(P, P \cap \mathbb{R}^{1,0}\right)=2, c_{\mathrm{LR}}\left(P_{1}, P_{1} \cap \mathbb{R}^{1,0}\right)=c_{\mathrm{LR}}\left(P_{2}, P_{2} \cap \mathbb{R}^{1,0}\right)=\frac{1}{2}$. Thus

$$
c_{\mathrm{LR}}\left(P, P \cap \mathbb{R}^{1,0}\right)>c_{\mathrm{LR}}\left(P_{1}, P_{1} \cap \mathbb{R}^{1,0}\right)+c_{\mathrm{LR}}\left(P_{2}, P_{2} \cap \mathbb{R}^{1,0}\right) .
$$

Moreover, for any $t \in(-1,1)$, the line $L_{t}:=\{(t, y) \mid y \in \mathbb{R}\}$ divides $P$ into two parts

$$
P_{+}:=\{(x, y) \in P \mid x \geq t\} \quad \text { and } \quad P_{-}:=\{(x, y) \in P \mid x \leq t\} .
$$

It is easily computed that

$$
c_{\mathrm{LR}}\left(P_{+}, P_{+} \cap \mathbb{R}^{1,0}\right)=1-t \quad \text { and } \quad c_{\mathrm{LR}}\left(P_{-}, P_{-} \cap \mathbb{R}^{1,0}\right)=1+t,
$$

and hence $c_{\mathrm{LR}}\left(P_{+}, P_{+} \cap \mathbb{R}^{1,0}\right)+c_{\mathrm{LR}}\left(P_{-}, P_{-} \cap \mathbb{R}^{1,0}\right)=c_{\mathrm{LR}}\left(P, P \cap \mathbb{R}^{1,0}\right)$.

In higher dimensions, we have $c_{\mathrm{LR}}\left(G, G \cap \mathbb{R}^{n, n}\right)=c_{\mathrm{EHZ}}(G)$ for any nonempty convex domain $G \subset \mathbb{R}^{2 n}$. Thus some coisotropic Hofer-Zehnder capacities of higher dimensions have subadditivity because of the subadditivity of $c_{\mathrm{EHZ}}$ under the conditions of [15, Theorem 1.8]. There is no nice result in more general case yet.

For the symmetrical Hofer-Zehnder symplectic capacity of a symmetric convex domain in $\mathbb{R}^{2 n}$ introduced by Liu and Wang [14, using a representation formula of it given by Rongrong Jin and the second named author in [9] one is able to generalize the formula in [15], but this is outside the scope of this paper and would appear elsewhere.

This paper is organized as follows. In the next section we collect detailed conclusions coming from [10, §4.1] and [11, §3.1] about proofs of representation formulas of the $\Psi$-EkelandHofer-Zehnder capacity and the coisotropic Ekeland-Hofer-Zehnder capacity for convex bodies in $\mathbb{R}^{2 n}$, respectively. Then we generalize some results on piecewise affine loops in [15, §3] to piecewise affine paths in Section 3. Theorem 1.1 will be proved in Section 4 Finally, in Section 5 we prove Theorems $1.2,1.3$. 


\section{Preliminaries}

For simplicity of the reader's convenience we list two results, which come from [10, Section 4.1] and [11, Section 3.1], respectively.

Let $K \subset \mathbb{R}^{2 n}$ be a compact convex domain $K$ with boundary $\mathcal{S}=\partial K$ and with $0 \in \operatorname{Int}(K)$. Denote by $H_{K}=\left(j_{K}\right)^{2}$ the square of the Minkowski functional $j_{K}$ of $K$, and by $H_{K}^{*}$ the Legendre transformation of $H_{K}$ defined by

$$
H_{K}^{*}(w)=\max _{\xi \in \mathbb{R}^{2 n}}\left(\langle x, \xi\rangle-H_{K}(\xi)\right) .
$$

Then $h_{K}^{2}=4 H_{K}^{*}$ (see e.g. [1]).

Given $\Psi \in \operatorname{Sp}(2 n, \mathbb{R})$ let $E_{\Psi}$ be the orthogonal complement of $\operatorname{Ker}\left(\Psi-I_{2 n}\right) \subset \mathbb{R}^{2 n}$ with respect to the standard inner product in $\mathbb{R}^{2 n}$. (In [10] we wrote $\operatorname{Ker}\left(\Psi-I_{2 n}\right)$ and $E_{\Psi}$ as $E_{1}$ and $E_{1}^{\perp}$, respectively.) Define

$$
\mathcal{F}_{\Psi}=\left\{x \in W^{1,2}\left([0,1], \mathbb{R}^{2 n}\right) \mid x(1)=\Psi x(0) \text { and } x(0) \in E_{\Psi}\right\},
$$

which was denoted by $\mathcal{F}$ in [10. If $\operatorname{dim} E_{\Psi}=0$, the problem reduces to the periodic case. So we only consider the non-periodic case in which $\operatorname{dim} E_{\Psi} \geqslant 1$. Define

$$
\mathcal{A}_{\Psi}=\left\{x \in \mathcal{F}_{\Psi} \mid A(x)=1\right\}
$$

where $A(x)$ is defined by (1.2) with $T=1$, and

$$
I_{K}: \mathcal{F}_{\Psi} \rightarrow \mathbb{R}, x \mapsto \int_{0}^{1} H_{K}^{*}(-J \dot{x}) .
$$

By Theorems 1.8, 1.9, Remark 1.10 and arguments in [10, §4.1] we have

Theorem 2.1. Under the above assumptions, $I_{K}$ attains its minimum $\min _{x \in \mathcal{A}_{\Psi}} I_{K}(x)$ over $\mathcal{A}_{\Psi}$, which is positive. For each minimier $u$ of $I_{K}$ over $\mathcal{A}_{\Psi}$, there exists $a_{0} \in \operatorname{Ker}\left(\Psi-I_{2 n}\right)$ such that the $W^{1,2}$-path

$$
\left[0, I_{K}(u)\right] \ni t \mapsto x^{*}(t)=\sqrt{I_{K}(u)} u\left(t / I_{K}(u)\right)+a_{0} / \sqrt{I_{K}(u)}
$$

satifies $A\left(x^{*}\right)=I_{K}(u)=c_{\mathrm{EHZ}}^{\Psi}(K)$ and

$$
\left\{\begin{array}{l}
-J \dot{x}^{*}(t) \in \partial H_{K}\left(x^{*}(t)\right), \text { a.e. } \\
x^{*}(T)=\Psi x^{*}(0) \quad \text { and } \quad x^{*}([0, T]) \subset \partial K
\end{array}\right.
$$

in particular $x^{*}$ is a generalized $\Psi$-characteristic on $\partial K$ because

$$
\partial H_{K}(x)=\left\{v \in N_{\partial K}(x) \mid\langle x, v\rangle=2\right\} \quad \forall x \in \partial K .
$$

(cf. Lemma 2 of [5, Chap.V, §1]). Conversely, if $z:[0, T] \rightarrow \partial K$ is a generalized $\Psi$ characteristic on $\partial K$ with action $A(z)=c_{\mathrm{EHZ}}^{\Psi}(K)$, then (by [10, Lemma 4.2]) there is a differentiable homeomorphism $\varphi:[0, T] \rightarrow[0, T]$ with an absolutely continuous inverse $\psi:$ $[0, T] \rightarrow[0, T]$ such that $z^{*}=z \circ \varphi$ is a $W^{1, \infty}{ }_{-}$map with action $A\left(z^{*}\right)=A(z)=T$ and satisfying (2.9); moreover we can choose $b \in \operatorname{Ker}\left(\Psi-I_{2 n}\right)$ so that the path $u:[0,1] \rightarrow \mathbb{R}^{2 n}$ defined by $u(t)=z^{*}(T t) / \sqrt{T}+b$ belongs to $\mathcal{A}_{\Psi}$ and satisfies $I_{K}(u)=T$, i.e., $u$ is a minimier $u$ of $I_{K}$ over $\mathcal{A}_{\Psi}$. When this $K$ is also a convex polytope as above (1.3), then there holds

$$
\dot{u}(t)=\sqrt{T} \dot{z}^{*}(T t) \in \sqrt{T} \operatorname{conv}\left\{p_{i} \mid \sqrt{T}(u(t)-b) \in F_{i}\right\}, \text { a.e. }
$$

where $p_{i}=\frac{2}{h_{i}} J n_{i}$. 
In order to see the final claim, note that for each $i=1, \cdots, \mathbf{F}_{K}, H_{K}$ is smooth at each relative interior point $x$ of $F_{i}$ and the subdifferential $\partial H_{K}(x)=\left\{\nabla H_{K}(x)\right\}=\left\{\frac{2}{h_{i}} n_{i}\right\}$. For any $x \in \partial K$ we have $\partial H_{K}(x)=\operatorname{conv}\left\{\frac{2}{h_{i}} n_{i} \mid x \in F_{i}\right\}$ (cf. [15, page 445]), and therefore $J \partial H_{K}(x)=\operatorname{conv}\left\{p_{i} \mid x \in F_{i}\right\}$. (The outward normal cone of $K$ at $x \in \partial K, N_{\partial K}(x)$, is equal to $\mathbb{R}_{+} \operatorname{conv}\left\{n_{i}: x \in F_{i}\right\}$.)

Fix an integer $0 \leq k<n$. Following [11] consider the Hilbert subspace of $W^{1,2}\left([0,1], \mathbb{R}^{2 n}\right)$,

$$
\mathcal{F}_{2}:=\left\{x \in W^{1,2}\left([0,1], \mathbb{R}^{2 n}\right) \mid x(0), x(1) \in \mathbb{R}^{n, k}, x(1) \sim x(0), \int_{0}^{1} x(t) d t \in J V_{0}^{n, k}\right\}
$$

(where $x(1) \sim x(0)$ means $x(1)-x(0) \in V_{0}^{n, k}$ ), its subset $\mathcal{A}_{2}=\left\{x \in \mathcal{F}_{2} \mid A(x)=1\right\}$, and the related convex functional

$$
I_{2}: \mathcal{F}_{2} \rightarrow \mathbb{R}, x \mapsto \int_{0}^{1} H_{K}^{*}(-J \dot{x}(t)) d t .
$$

From [11, §3.1], we obtain the following corresponding result of Theorem 2.1]

Theorem 2.2. Under the above assumptions, $I_{2}$ attains its minimum $\min _{x \in \mathcal{A}_{2}} I_{2}(x)$ over $\mathcal{A}_{2}$, which is positive. For each minimier $u$ of $I_{2}$ over $\mathcal{A}_{2}$, there exists $\mathbf{a}_{0} \in \mathbb{R}^{n, k}$ such that the $W^{1,2}$-path

$$
[0,1] \ni t \mapsto x^{*}(t):=\sqrt{I_{2}(u)} u(t)+\mathbf{a}_{0} / \sqrt{I_{2}(u)}
$$

satisfies $A\left(x^{*}\right)=I_{2}(u)=c_{\mathrm{LR}}\left(K, K \cap \mathbb{R}^{n, k}\right)=c^{n, k}(K)$ and

$$
\left\{\begin{array}{l}
-J \dot{x}^{*}(t)=\partial H_{K}\left(x^{*}(t)\right), \text { a.e., } x^{*}(0), x^{*}(1) \in \mathbb{R}^{n, k}, \\
x^{*}(1)-x^{*}(0) \in V_{0}^{n, k} \quad \text { and } \quad x^{*}([0,1]) \subset \partial K
\end{array}\right.
$$

in particular $x^{*}$ is a generalized leafwise chord on $\partial K$ for $\mathbb{R}^{n, k}$ because of (2.10). Conversely, if $z:[0, T] \rightarrow \partial K$ is a generalized leafwise chord on $\partial K$ with action $A(z)=c^{n, k}(K)$ for $\mathbb{R}^{n, k}$, then (by [10, Lemma 4.2]) there is a differentiable homeomorphism $\varphi:[0, T] \rightarrow[0, T]$ with an absolutely continuous inverse $\psi:[0, T] \rightarrow[0, T]$ such that $z^{*}=z \circ \varphi$ is a $W^{1, \infty}$-map with action $A\left(z^{*}\right)=A(z)=T$ and satisfying

$$
\left\{\begin{array}{l}
-J \dot{z}^{*}(t)=\partial H_{K}\left(z^{*}(t)\right), \text { a.e., } z^{*}(0), z^{*}(T) \in \mathbb{R}^{n, k}, \\
z^{*}(T)-z^{*}(0) \in V_{0}^{n, k} \text { and } z^{*}([0, T]) \subset \partial K
\end{array}\right.
$$

moreover the path $u:[0,1] \rightarrow \mathbb{R}^{2 n}$ defined by

$$
u(t)=\frac{1}{\sqrt{T}} z^{*}(T t)-\frac{1}{\sqrt{T}} P_{n, k} \int_{0}^{1} z^{*}(T t) d t
$$

where $P_{n, k}: \mathbb{R}^{2 n}=J V_{0}^{n, k} \oplus \mathbb{R}^{n, k} \rightarrow \mathbb{R}^{n, k}$ is the orthogonal projection, belongs to $\mathcal{A}_{2}$ and satisfies $I_{2}(u)=T$, i.e., $u$ is a minimier $u$ of $I_{2}$ over $\mathcal{A}_{2}$. When this $K$ is also a convex polytope as above (1.3), there holds

$$
\dot{u}(t)=\sqrt{T} \dot{z}^{*}(T t) \in \sqrt{T} \operatorname{conv}\left\{p_{i} \mid \sqrt{T}(u(t)-b) \in F_{i}\right\}, \text { a.e. }
$$

where $p_{i}=\frac{2}{h_{i}} J n_{i}$ and $b=-\frac{1}{\sqrt{T}} P_{n, k} \int_{0}^{1} z^{*}(T t) d t$.

The final claim is obtained as below Theorem 2.1. 


\section{$3 \quad$ Piecewise affine paths}

In this section we will generalize some results on piecewise affine loops in [15, §3] to piecewise affine paths.

Recall in [15, Definition 3.2] that a finite sequence of disjoint open intervals $\left(I_{i}\right)_{i=1}^{m}$ is called a partition of $[0,1]$ if there exists an increasing sequence of numbers $0=\tau_{0} \leq \tau_{1} \leq \ldots \leq \tau_{m}=1$ with $I_{i}=\left(\tau_{i-1}, \tau_{i}\right)$. (Note that the open interval $I_{i}$ may be empty!) As usual let $\chi_{I}$ denote the characteristic function of a subset $I \subset \mathbb{R}$. A path $z \in H^{1}\left([0,1], \mathbb{R}^{2 n}\right)$ is said to be piecewise affine if $\dot{z}$ can be written as $\dot{z}(t)=\sum_{j=1}^{m} \chi_{I_{j}}(t) w_{j}$ for almost every $t \in[0,1]$, where $\left(I_{j}\right)_{j=1}^{m}$ is a partition of $[0,1]$ and $\left(w_{j}\right)_{j=1}^{m} \in \mathbb{R}^{2 n}$ is a finite sequence of vectors.

Lemma 3.1 ([15, Lemma 3.1]). Fix a set of vectors $v_{1}, \cdots, v_{k} \in \mathbb{R}^{2 n}$. Suppose $z \in H^{1}\left([0,1], \mathbb{R}^{2 n}\right)$ satisfies that for almost every $t \in[0,1]$, one has $\dot{z}(t) \in \operatorname{conv}\left\{v_{1}, \cdots, v_{k}\right\}$. Then for every $\varepsilon>0$, there exists a piecewise affine path $\varsigma$ with $\|z-\varsigma\|_{W^{1,2}}<\varepsilon$, and so that $\dot{\varsigma}$ is composed of vectors from the set $\operatorname{conv}\left\{v_{1}, \cdots, v_{k}\right\}$, and $\varsigma(0)=z(0), \varsigma(1)=z(1)$.

The following is an analouge of [15, Proposition 3.3].

Proposition 3.2. If a path $z \in H^{1}\left([0,1], \mathbb{R}^{2 n}\right)$ is such that $\dot{z}(t)=\sum_{i=1}^{m} \chi_{I_{i}}(t) w_{i}$ almost everywhere, where $\left(I_{i}=\left(\tau_{i-1}, \tau_{i}\right)\right)_{i=1}^{m}$ is a partition of $[0,1]$, and $w_{1}, \cdots, w_{m} \in \mathbb{R}^{2 n}$, then

$$
\int_{0}^{1}\langle-J \dot{z}, z\rangle d t=\sum_{i=1}^{m} \sum_{j=1}^{i-1}\left|I_{j}\right|\left|I_{i}\right| \omega_{0}\left(w_{j}, w_{i}\right)+\omega_{0}(z(0), z(1)) .
$$

As usual $\sum_{j=1}^{i-1}\left|I_{j}\right|\left|I_{i}\right| \omega_{0}\left(w_{j}, w_{i}\right)$ for $i=1$ is understood as zero.

Proof. The case $m=1$ is clear. Now we assume $m>1$. Since

$$
\int_{0}^{1}\langle-J \dot{z}(t), z(0)\rangle d t=-\langle J z(1), z(0)\rangle=-\omega_{0}(J z(1), J z(0))=\omega_{0}(z(0), z(1))
$$

we deduce

$$
\begin{aligned}
\int_{0}^{1}\langle-J \dot{z}, z\rangle d t= & \int_{0}^{1}\left\langle-J \dot{z}, z(0)+\int_{0}^{t} \dot{z}(s) d s\right\rangle d t \\
= & \int_{0}^{1}\langle-J \dot{z}(t), z(0)\rangle d t \\
& +\sum_{i=1}^{m} \int_{I_{i}}\left\langle-J \sum_{l=1}^{m} \chi_{I_{l}}(t) w_{l}, \int_{0}^{\tau_{i-1}} \sum_{l=1}^{m} \chi_{I_{l}}(s) w_{l} d s+\int_{\tau_{i-1}}^{t} w_{i} d s\right\rangle d t \\
= & \omega_{0}(z(0), z(1))+\sum_{i=1}^{m} \int_{I_{i}}\left\langle-J w_{i}, \sum_{j<i} \int_{I_{j}} \sum_{l=1}^{m} \chi_{I_{l}}(s) w_{l} d s+\left(t-\tau_{i-1}\right) w_{i}\right\rangle d t \\
= & \omega_{0}(z(0), z(1))+\sum_{i=1}^{m} \int_{I_{i}}\left\langle-J w_{i}, \sum_{j<i} \int_{I_{j}} w_{j} d s\right\rangle d t \\
= & \omega_{0}(z(0), z(1))+\sum_{i=1}^{m} \sum_{j<i}\left|I_{i}\right|\left|I_{j}\right| \omega_{0}\left(w_{j}, w_{i}\right) .
\end{aligned}
$$

Following the proof ideas of [15, Lemma 3.1] we can obtain: 
Lemma 3.3. Given a set of vectors, $v_{1}, \ldots, v_{k} \in \mathbb{R}^{2 n}$, for any piecewise affine path $z \in$ $H^{1}\left([0,1], \mathbb{R}^{2 n}\right)$ with $\dot{z}(t) \in \operatorname{conv}\left\{v_{1}, \ldots, v_{k}\right\}$ for almost every $t \in[0,1]$, there exists another piecewise affine path $z^{\prime} \in H^{1}\left([0,1], \mathbb{R}^{2 n}\right)$ so that $z^{\prime}(0)=z(0), z^{\prime}(1)=z(1), \dot{z}^{\prime}(t) \in\left\{v_{1}, \ldots, v_{k}\right\}$ for almost every $t$, and

$$
\int_{0}^{1}\left\langle-J \dot{z}^{\prime}, z^{\prime}\right\rangle d t \geq \int_{0}^{1}\langle-J \dot{z}, z\rangle d t
$$

Proof. Write $\dot{z}(t)=\sum_{j=1}^{m} \chi_{I_{j}}(t) w_{j}$, where $w_{j} \in \operatorname{conv}\left\{v_{1}, \ldots, v_{k}\right\}$ for each $j$, and $\left(I_{j}\right)_{j=1}^{m}$ is a partition of $[0,1]$. Clearly, there exists $l=l(i) \in \mathbb{N}$ such that $w_{i}=\sum_{j=1}^{l} a_{i_{j}} v_{i_{j}}$, where $a_{i_{j}}>0, i_{j} \in\{1, \ldots, k\}$, and $\sum_{j=1}^{l} a_{i_{j}}=1$. Consider the partition of $I_{i}$ to disjoint subintervals, $\left\{I_{i_{j}}\right\}_{j=1}^{l}$, where the length of $I_{i_{j}}$ is $\left|I_{i_{j}}\right|=a_{i_{j}}\left|I_{i}\right|$. Define

$$
\dot{z}_{*}(t)=\sum_{j<i} \chi_{I_{j}}(t) w_{j}+\sum_{j=1}^{l} \chi_{I_{i_{j}}}(t) v_{i_{j}}+\sum_{j>i} \chi_{I_{j}}(t) w_{j}
$$

and $z_{*}(t)=z(0)+\int_{0}^{t} \dot{z}_{*}(s) d s$ for $t \in[0,1]$. Since $\int_{0}^{1} \dot{z}_{*}(t) d t=\int_{0}^{1} \dot{z}(t) d t$, we deduce $z(0)=z_{*}(0)$ and $z(1)=z_{*}(1)$. Then Proposition 3.2 leads to

$$
\begin{aligned}
\int_{0}^{1}\left\langle-J \dot{z}_{*}, z_{*}\right\rangle d t & =\omega_{0}\left(z_{*}(0), z_{*}(1)\right)+\sum_{\substack{r<s \\
r, s \neq i}}\left|I_{r}\right|\left|I_{s}\right| \omega_{0}\left(w_{r}, w_{s}\right)+\sum_{j=1}^{l} \sum_{r<i}\left|I_{r}\right|\left|I_{i}\right| a_{i_{j}} \omega_{0}\left(w_{r}, v_{i_{j}}\right) \\
& +\sum_{j=1}^{l} \sum_{r>i}\left|I_{r}\right|\left|I_{i}\right| a_{i_{j}} \omega_{0}\left(v_{i_{j}}, w_{r}\right)+\sum_{1 \leq r<s \leq l}\left|I_{i}\right|^{2} a_{i_{r}} a_{i_{s}} \omega_{0}\left(v_{i_{r}}, v_{i_{s}}\right) \\
& =\omega_{0}(z(0), z(1))+\sum_{\substack{r<s \\
r, s \neq i}}\left|I_{r}\right|\left|I_{s}\right| \omega_{0}\left(w_{r}, w_{s}\right)+\sum_{r<i}\left|I_{r}\right|\left|I_{i}\right| \omega_{0}\left(w_{r}, w_{i}\right) \\
& +\sum_{r>i}\left|I_{r}\right|\left|I_{i}\right| \omega_{0}\left(w_{i}, w_{r}\right)+\sum_{1 \leq r<s \leq l}\left|I_{i}\right|^{2} a_{i_{r}} a_{i_{s}} \omega_{0}\left(v_{i_{r}}, v_{i_{s}}\right) \\
& =\int_{0}^{1}\langle-J \dot{z}, z\rangle d t+\left|I_{i}\right|^{2} \sum_{1 \leq r<s \leq l} a_{i_{r}} a_{i_{s}} \omega_{0}\left(v_{i_{r}}, v_{i_{s}}\right) .
\end{aligned}
$$

Define $b_{i_{j}}=a_{i_{l+1-j}}$ and $u_{i_{j}}=v_{i_{l+1-j}}$ for $j=1, \cdots, l$, and

$$
\hat{I}_{j}=I_{j} \text { for } j<i \text { or } j>i, \quad \hat{I}_{i_{j}}=I_{i_{l+1-j}} \text { for } j=1, \cdots, l .
$$

As above we may show that $z_{* *}(t)=z(0)+\int_{0}^{t} \dot{z}_{* *}(s) d s$ for $t \in[0,1]$, where

$$
\dot{z}_{* *}(t)=\sum_{j<i} \chi_{\hat{I}_{j}}(t) w_{j}+\sum_{j=1}^{l} \chi_{\hat{I}_{i_{j}}}(t) u_{i_{j}}+\sum_{j>i} \chi_{\hat{I}_{j}}(t) w_{j},
$$

satisfies $z(0)=z_{* *}(0), z(1)=z_{* *}(1)$ and

$$
\int_{0}^{1}\left\langle-J \dot{z}_{* *}, z_{* *}\right\rangle d t=\int_{0}^{1}\langle-J \dot{z}, z\rangle d t+\left|I_{i}\right|^{2} \sum_{1 \leq r<s \leq l} b_{i_{r}} b_{i_{s}} \omega_{0}\left(u_{i_{r}}, u_{i_{s}}\right) .
$$

A straightforward computation as above gives rise to

$$
\sum_{1 \leq r<s \leq l} b_{i_{r}} b_{i_{s}} \omega_{0}\left(u_{i_{r}}, u_{i_{s}}\right)=-\sum_{1 \leq r<s \leq l} a_{i_{r}} a_{i_{s}} \omega_{0}\left(v_{i_{r}}, v_{i_{s}}\right) .
$$


Hence we can always choose $u \in\left\{z_{*}, z_{* *}\right\}$ so that

$$
\int_{0}^{1}\langle-J \dot{u}, u\rangle d t \geq \int_{0}^{1}\langle-J \dot{z}, z\rangle d t
$$

Now starting from $z$ and choosing $i=1$ we get a path $z_{1}$ as above, Then starting from $z_{1}$ and choosing $i=2$ we get a path $z_{2}$ again. Continuing this progress we obtain $z_{1}, z_{2}, \cdots, z_{m}$. Then $z^{\prime}:=z_{m}$ satisfies the requirements of the lemma.

Suitably modifying the proof of [15, Lemma 3.5], we can get the following analogues of it.

Lemma 3.4. Given a finite sequence of pairwise distinct vectors $\left(v_{1}, \cdots, v_{k}\right)$, if $z \in H^{1}\left([0,1], \mathbb{R}^{2 n}\right)$ is a piecewise affine path such that $\dot{z}(t)=\sum_{i=1}^{m} \chi_{I_{i}}(t) w_{i}$ with $w_{i} \in\left\{v_{1}, \cdots, v_{k}\right\}$ for each $i$, where $\left(I_{i}=\left(\tau_{i-1}, \tau_{i}\right)\right)_{i=1}^{m}$ is a partition of $[0,1]$, then there exists another piecewise affine path $z^{\prime}$ such that $\dot{z}^{\prime}(t) \in\left\{v_{1}, \cdots, v_{k}\right\}$ for almost every $t, z^{\prime}(0)=z(0), z^{\prime}(1)=z(1)$, and $\left\{t: \dot{z}^{\prime}(t)=v_{j}\right\}$ is connected for every $j=1, \cdots, k$. In addition,

$$
\int_{0}^{1}\left\langle-J \dot{z}^{\prime}, z^{\prime}\right\rangle d t \geq \int_{0}^{1}\langle-J \dot{z}, z\rangle d t
$$

Proof. Assume $w_{r}=w_{s}$ for some $r<s$. Consider a rearrangement of the intervals $I_{i}$ by deleting the intervals $I_{s}$ and increasing the length of the interval $I_{r}$ by $\left|I_{s}\right|=\tau_{s}-\tau_{s-1}$, that is,

$$
I_{i}^{*}=\left\{\begin{array}{lr}
\left(\tau_{i-1}, \tau_{i}\right), & i<r, \\
\left(\tau_{i-1}, \tau_{i}+\tau_{s}-\tau_{s-1}\right), & i=r, \\
\left(\tau_{i-1}+\tau_{s}-\tau_{s-1}, \tau_{i}+\tau_{s}-\tau_{s-1}\right), & r<i<s, \\
\emptyset, & i=s, \\
\left(\tau_{i-1}, \tau_{i}\right), & i>s .
\end{array}\right.
$$

Define $z_{*}$ by $z_{*}(t)=z(0)+\int_{0}^{t} \dot{z}_{*}(s) d s$, where $\dot{z}_{*}(t)=\sum_{i=1}^{m} \chi_{I_{i}^{*}}(t) w_{i}$. Then

$$
\int_{0}^{1} \dot{z}_{*} d t=\sum_{i=1}^{m}\left|I_{i}^{*}\right| w_{i}=\sum_{i=1}^{m}\left|I_{i}\right| w_{i}=\int_{0}^{1} \dot{z} d t
$$

and thus $z_{*}(0)=z(0)$ and $z_{*}(1)=z(1)$. Since $I_{i}^{*}=I_{i}$ for $i<r$ or $i>s$, by Proposition 3.2 , one can get

$$
\int_{0}^{1}\left\langle-J \dot{z}_{*}, z_{*}\right\rangle d t-\int_{0}^{1}\langle-J \dot{z}, z\rangle d t=\sum_{i=r+1}^{s-1} 2\left|I_{s}\right|\left|I_{i}\right| \omega_{0}\left(w_{s}, w_{i}\right) .
$$

Similarly, by erasing $I_{r}$ and increasing the length of $I_{s}$ by $\left|I_{r}\right|$, we get a $z_{* *}$ such that

$$
\int_{0}^{1}\left\langle-J \dot{z}_{* *}, z_{* *}\right\rangle d t-\int_{0}^{1}\langle-J \dot{z}, z\rangle d t=\sum_{i=r+1}^{s-1} 2\left|I_{r}\right|\left|I_{i}\right| \omega_{0}\left(w_{i}, w_{r}\right) .
$$

It follows that either $z_{*}$ or $z_{* *}$ satisfies (3.19). Denote by $z_{1} \in\left\{z_{*}, z_{* *}\right\}$ satisfying (3.19). Then

$$
z_{1}(t)=z(0)+\int_{0}^{t} \dot{z}_{1}(s) d s \quad \text { with } \dot{z}_{1}(t)=\sum_{i=1}^{m} \chi_{I_{i}^{1}}(t) w_{i}
$$

Repeating this methods for different disjoint nonempty interval $I_{r}^{1}, I_{s}^{1}$ whenever $w_{r}=w_{s}$ we get a $z_{2}$ again. Proceeding with this progress for $z_{2}$, after finite steps we get a $z^{\prime}$ with the expected properties. 
Having the above lemmas we have the following corresponding result with [15, Proposition 3.5], which may be proved by repeating the arguments therein because $H_{K}^{*}=\frac{1}{4} h_{K}^{2}$.

Proposition 3.5. For a convex polytope $K \subset \mathbb{R}^{2 n}$ containing 0 in the interior of it, let $\left\{F_{i}\right\}_{i=1}^{\mathbf{F}_{K}}$ be the $(2 n-1)$-dimensional facets of it, let $n_{i}$ be the unit outer normal to $F_{i}$, let $p_{i}=\left.J \partial H_{K}\right|_{F_{i}}=\frac{2}{h_{i}} J n_{i}$, where $h_{i}:=h_{K}\left(n_{i}\right)$ and $h_{K}(x)=\sup \{\langle y, x\rangle \mid y \in K\}$. Let $c>0$ be $a$ constant and let $z \in H^{1}\left([0,1], \mathbb{R}^{2 n}\right)$ satisfies that for almost every $t$, there is a non-empty face of $K, F_{j_{1}} \cap \cdots \cap F_{j_{l}} \neq \emptyset$, with $\dot{z}(t) \in c \cdot \operatorname{conv}\left\{p_{j_{1}}, \cdots, p_{j_{l}}\right\}$. Then

$$
\int_{0}^{1} H_{K}^{*}(-J \dot{z}(t)) d t=c^{2}
$$

\section{Proof of Theorem 1.1}

We begin with a similar result to [15, Theorem 1.5].

Theorem 4.1. Let $K$ be a convex polytope as above (1.3). Suppose $0 \in \operatorname{Int}(K)$. Then for any $\Psi \in \operatorname{Sp}(2 n, \mathbb{R})$ there exists a generalized $\Psi$-characteristic $\gamma:[0,1] \rightarrow \partial K$ with action

$$
A(\gamma)=\min \{A(x)>0 \mid x \text { is a generalized } \Psi \text {-characteristic on } \partial K\}
$$

such that $\dot{\gamma}$ is piecewise constant and is composed of a finite sequence of vectors, i.e. there exists a sequence of vectors $\left(w_{1}, \ldots, w_{m}\right)$, and a sequence $\left(0=\tau_{0}<\cdots<\tau_{m-1}<\tau_{m}=1\right)$ so that $\dot{\gamma}(t)=w_{i}$ for $\tau_{i-1}<t<\tau_{i}$. Moreover, for each $j \in\{1, \cdots, m\}$ there exists $i \in\left\{1, \cdots, \mathbf{F}_{K}\right\}$ so that $w_{j}=C_{j} J n_{i}$ for some $C_{j}>0$, and for each $i \in\left\{1, \cdots, \mathbf{F}_{K}\right\}$ and for every $C>0$ the set $\left\{t \in[0,1] \mid \dot{\gamma}(t)=C J n_{i}\right\}$ is either empty or connected, i.e. for every $i$ there is at most one $j \in\{1, \ldots, m\}$ with $w_{j}=C_{j} J n_{i}$. Hence $\dot{\gamma}$ has at most $\mathbf{F}_{K}$ discontinuous points, and $\gamma$ visits the interior of each facet at most once.

Proof. Let $z:[0, T] \rightarrow \partial K$ be a generalized $\Psi$-characteristic with action $A(z)=c_{\mathrm{EHz}}^{\Psi}(K)=T$. By Theorem 2.1 we have $b \in \operatorname{Ker}\left(\Psi-I_{2 n}\right)$ and the $W^{1,2}$-path $u \in \mathcal{A}_{\Psi}$ satisfying $I_{K}(u)=T$ and (2.11). Thus we obtain $\int_{0}^{1} H_{K}^{*}(-J \dot{u}(t)) d t=T$ by Proposition 3.5. For convenience let $c=T^{1 / 2}$. The next argument is the same as the proof of [15, Theorem 1.5], we write it for completeness.

For every $N \in \mathbb{N}$, Lemma 3.1 yields a piecewise affine path $\zeta_{N}$ such that

$$
\left\|u-\zeta_{N}\right\|_{W^{1,2}} \leqslant \frac{1}{N} \quad \text { and } \quad \dot{\zeta}_{N}(t) \in c \cdot \operatorname{conv}\left\{p_{1}, \cdots, p_{\mathbf{F}_{K}}\right\}
$$

for almost every $t, \zeta_{N}(0)=u(0), \zeta_{N}(1)=u(1)$. By applying Lemma 3.3 with $v_{i}=c p_{i}, i=$ $1, \cdots, \mathbf{F}_{K}$ to $\zeta_{N}$, we get a piecewise affine path $\zeta_{N}^{\prime} \in W^{1,2}\left([0,1], \mathbb{R}^{2 n}\right)$ such that

$$
\zeta_{N}^{\prime}(0)=u(0), \quad \zeta_{N}^{\prime}(1)=u(1), \quad \dot{\zeta}_{N}^{\prime}(t) \in\left\{v_{1}, \ldots, v_{\mathbf{F}_{K}}\right\} \text { a.e., and } A\left(\zeta_{N}^{\prime}\right) \geq A\left(\zeta_{N}\right) .
$$

Applying Lemma 3.4 to $\zeta_{N}^{\prime}$ again, we get a piecewise affine path $u_{N}:[0,1] \rightarrow \mathbb{R}^{2 n}$ from $u(0)$ to $u(1)$ such that

$$
\dot{u}_{N}(t)=\sum_{i=1}^{m_{N}} \chi_{I_{i}^{N}}(t) v_{i}^{N}
$$

where $v_{i}^{N}=v_{j}$ for some $j \in\left\{1, \cdots, \mathbf{F}_{K}\right\}$ and for every $j$ there is at most one such $i$, and that

$$
A_{N}:=\sqrt{A\left(u_{N}\right)} \geqslant \sqrt{A\left(\zeta_{N}\right)}
$$

Define $u_{N}^{\prime}:=\frac{u_{N}}{A_{N}} \in \mathcal{A}_{\Psi}$ and $c_{N}=: \frac{c}{A_{N}}$. Write $w_{i}^{N}:=\frac{v_{i}^{N}}{A_{N}}$ for the velocities of $u_{N}^{\prime}$, which sits in the set $\frac{c}{A_{N}} \cdot\left\{p_{1}, \cdots, p_{\mathbf{F}_{K}}\right\}$. Since $\left\|u-\zeta_{N}\right\|_{W^{1,2}} \leqslant \frac{1}{N}$ we deduce that $A\left(\zeta_{N}\right) \rightarrow 1$ as 
$N \rightarrow \infty$. Hence $\underline{\lim }_{N \rightarrow \infty} A_{N} \geqslant 1$, and $\overline{\lim }_{N \rightarrow \infty} c_{N} \leqslant c$. Moreover Proposition 3.5 and the minimality of $I_{K}(u)$ imply $c_{N}^{2}=I_{K}\left(u_{N}^{\prime}\right) \geqslant I_{K}(u)=c^{2}$. We deduce $\lim _{N \rightarrow \infty} c_{N}=c$ and thus $\lim _{N \rightarrow \infty} A_{N}=1$.

Let $\mathcal{A}^{1}$ consist of $z \in H^{1}\left([0,1], \mathbb{R}^{2 n}\right)$ for which there exist $C>0$ and an increasing sequence of numbers $0=\tau_{0} \leq \tau_{1} \leq \ldots \leq \tau_{\mathbf{F}_{K}}=1$ such that

$$
\dot{z}(t)=\sum_{i=1}^{\mathbf{F}_{K}} \chi_{I_{i}}(t) C \cdot p_{\sigma(i)}
$$

with $I_{i}=\left(\tau_{i-1}, \tau_{i}\right)$, where $\sigma \in S_{\mathbf{F}_{K}}$ is the permutations on $\left\{1, \cdots, \mathbf{F}_{K}\right\}$. Define a map

$$
\Phi: \mathcal{A}^{1} \rightarrow S_{\mathbf{F}_{K}} \times \mathbb{R}^{\mathbf{F}_{K}}, z \mapsto\left(\sigma,\left(\left|I_{1}\right|, \cdots,\left|I_{\mathbf{F}_{K}}\right|\right)\right) .
$$

Clearly, the image $\operatorname{Im}(\Phi)$ is contained in the compact subset of $S_{\mathbf{F}_{K}} \times \mathbb{R}^{\mathbf{F}_{K}}$,

$$
S_{\mathbf{F}_{K}} \times\left\{\left(t_{1}, \cdots, t_{\mathbf{F}_{K}}\right) \in \mathbb{R}^{\mathbf{F}_{K}} \mid t_{i} \geqslant 0 \forall i, \sum_{i=1}^{\mathbf{F}_{K}} t_{i}=1\right\} .
$$

Since $u_{N}^{\prime} \in \mathcal{A}^{1}$ with $C=c_{N}$, we can write $\Phi\left(u_{N}^{\prime}\right)=\left(\sigma^{N},\left(t_{1}^{N}, \cdots, t_{\mathbf{F}_{K}}^{N}\right)\right)$. After passing to a subsequence, we can assume that $\sigma^{N}=\sigma$ is constant, and $\left(t_{1}^{N}, \cdots, t_{\mathbf{F}_{K}}^{N}\right)$ converges to a vector $\left(t_{1}^{\infty}, \cdots, t_{\mathbf{F}_{K}}^{\infty}\right)$. Define

$$
\begin{aligned}
& \tau_{0}^{\infty}=0, \tau_{1}^{\infty}=\tau_{0}^{\infty}+t_{1}^{\infty}, \tau_{j}^{\infty}=\tau_{0}^{\infty}+\sum_{i=1}^{j} t_{i}^{\infty}, j=2, \cdots, \mathbf{F}_{K}, \\
& I_{i}^{\infty}=\left(\tau_{i-1}^{\infty}, \tau_{i}^{\infty}\right), i=1, \cdots, \mathbf{F}_{K}
\end{aligned}
$$

and the piecewise affine path $u_{\infty}^{\prime}(t):=u(0)+\int_{0}^{t} \dot{u}_{\infty}^{\prime}(s) d s$ with

$$
\dot{u}_{\infty}^{\prime}(t)=\sum_{i=1}^{\mathbf{F}_{K}} \chi_{I_{i}^{\infty}}(t) c \cdot p_{\sigma(i)} .
$$

Let $\mathcal{T}^{N}=\left\{t \in[0,1] \mid \dot{u}_{N}^{\prime}(t)=\frac{c}{c_{N}} \dot{u}_{\infty}^{\prime}(t)\right\}$. Then

$$
\int_{\mathcal{T}^{N}}\left\|\dot{u}_{N}^{\prime}(t)-\dot{u}_{\infty}^{\prime}(t)\right\|^{2} d t \rightarrow 0 \quad \text { as } N \rightarrow \infty .
$$

Since $\left\|\dot{u}_{N}^{\prime}(t)-\dot{u}_{\infty}^{\prime}(t)\right\|^{2}$ is bounded on $\left\{t \in[0,1] \mid \dot{z}_{N}^{\prime}(t)\right.$ and $\dot{z}_{\infty}^{\prime}(t)$ are defined $\}$, as $N \rightarrow \infty$ we get $\left|\mathcal{T}^{N}\right| \rightarrow 1$ and therefore

$$
\int_{[0,1] \backslash \mathcal{T}^{N}}\left\|\dot{u}_{N}^{\prime}(t)-\dot{u}_{\infty}^{\prime}(t)\right\|^{2} d t \rightarrow 0 .
$$

Observe that $\lim _{N \rightarrow \infty} \int_{0}^{1} \dot{u}_{N}^{\prime}(t) d t=\int_{0}^{1} \dot{u}(t) d t$ implies $\int_{0}^{1} \dot{u}_{\infty}^{\prime}(t) d t=\int_{0}^{1} \dot{u}(t) d t$. We deduce

$$
u_{\infty}^{\prime}(1)=u_{\infty}^{\prime}(0)+\int_{0}^{1} \dot{u}_{\infty}^{\prime}(t) d t=u(0)+\int_{0}^{1} \dot{u}(t) d t=u(1)
$$

and so $u_{\infty}^{\prime}(1)=\Psi u_{\infty}^{\prime}(0)$. Moreover

$$
\begin{aligned}
\left|A\left(u_{\infty}^{\prime}\right)-1\right| & =\left|A\left(u_{\infty}^{\prime}\right)-A\left(u_{N}^{\prime}\right)\right| \\
& =\left|\frac{1}{2} \int_{0}^{1}\left\langle-J \dot{u}_{\infty}^{\prime}(t), u_{\infty}^{\prime}(t)\right\rangle-\left\langle-J \dot{u}_{N}^{\prime}(t), u_{N}^{\prime}(t)\right\rangle d t\right| \\
& \leqslant\left|\frac{1}{2} \int_{0}^{1}\left\langle-J\left(\dot{u}_{\infty}^{\prime}(t)-\dot{u}_{N}^{\prime}(t)\right), u_{\infty}^{\prime}(t)\right\rangle d t\right|+\left|\frac{1}{2} \int_{0}^{1}\left\langle-J \dot{u}_{N}^{\prime}(t), u_{\infty}^{\prime}(t)-u_{N}^{\prime}(t)\right\rangle d t\right| \\
& \leqslant \frac{1}{2} \int_{0}^{1}\left|\dot{u}_{\infty}^{\prime}(t)-\dot{u}_{N}^{\prime}(t)\right|\left|u_{\infty}^{\prime}(t)\right| d t+\frac{1}{2} \int_{0}^{1}\left|\dot{u}_{N}^{\prime}(t)\right|\left|u_{\infty}^{\prime}(t)-u_{N}^{\prime}(t)\right| d t \rightarrow 0
\end{aligned}
$$


because $\dot{u}_{N}^{\prime}$ and $u_{\infty}^{\prime}$ are bounded. Then $A\left(u_{\infty}^{\prime}\right)=1$, and thus $u_{\infty}^{\prime} \in \mathcal{A}_{\Psi}$ and

$$
I_{K}\left(u_{\infty}^{\prime}\right)=\lim _{N \rightarrow \infty} I_{K}\left(u_{N}^{\prime}\right)=\lim _{N \rightarrow \infty} c_{N}^{2}=c^{2}=T=c_{\mathrm{EHZ}}^{\Psi}(K) .
$$

By Theorem 2.1 we have $a_{0} \in \operatorname{Ker}\left(\Psi-I_{2 n}\right)$ such that the $W^{1,2}$-path

$$
[0, T] \ni t \mapsto \gamma^{*}(t)=\sqrt{T} u_{\infty}^{\prime}(t / T)+a_{0} / \sqrt{T}
$$

is a piecewise affine generalized $\Psi$-characteristic on $\partial K$ with action $A\left(\gamma^{*}\right)=c_{\mathrm{EHZ}}^{\Psi}(K)$. Then the generalized $\Psi$-characteristic on $\partial K,[0,1] \ni t \mapsto \gamma(t):=\gamma^{*}(T t)$, has action $A(\gamma)=$ $c_{\mathrm{EHZ}}^{\Psi}(K)$ and satisfies $\dot{\gamma}(t) \in T \cdot\left\{p_{1}, \cdots, p_{\mathbf{F}_{K}}\right\}$ for almost every $t \in[0,1]$ and that the set $\left\{t: \dot{\gamma}(t)=p_{i}\right\}$ is connected for every $i$. Recall $p_{i}=\frac{2}{h_{i}} J n_{i}$. Theorem 4.1 is proved.

Proof of Theorem 1.1. Step 1. Case $0 \in \operatorname{Int}(K)$. Let $\mathcal{A}_{\Psi}^{0}$ consist of $z \in \mathcal{A}_{\Psi}$ for which there exist $C>0$ and an increasing sequence of numbers $0=\tau_{0} \leq \tau_{1} \leq \ldots \leq \tau_{\mathbf{F}_{K}}=1$ such that

$$
\dot{z}(t)=\sum_{i=1}^{\mathbf{F}_{K}} \chi_{I_{i}}(t) C \cdot p_{\sigma(i)}
$$

with $I_{i}=\left(\tau_{i-1}, \tau_{i}\right)$, where $\sigma \in S_{\mathbf{F}_{K}}$ is a permutation on $\left\{1, \cdots, \mathbf{F}_{K}\right\}$. Then $u_{\infty}^{\prime}$ in the proof of Theorem 4.1 belongs to $\mathcal{A}_{\Psi}^{0}$ and satisfies $I_{K}\left(u_{\infty}^{\prime}\right)=c_{\mathrm{EHZ}}^{\Psi}(K)$. Thus

$$
c_{\mathrm{EHZ}}^{\Psi}(K)=\min \left\{I_{K}(z) \mid z \in \mathcal{A}_{\Psi}\right\}=\min \left\{I_{K}(z) \mid z \in \mathcal{A}_{\Psi}^{0}\right\} .
$$

For any $z \in \mathcal{A}_{\Psi}^{0}, \dot{z}$ has the form of (4.22) and hence

$$
z(1)-z(0)=\int_{0}^{1} \dot{z}(t) d t=C \sum_{i=1}^{\mathbf{F}_{K}} T_{i} p_{\sigma(i)}
$$

where $T_{i}=\left|I_{i}\right|$, and Proposition 3.2 yields

$$
1=\frac{1}{2} \int_{0}^{1}\langle-J \dot{z}, z\rangle d t=\frac{1}{2} C^{2} \sum_{1 \leqslant j<i \leqslant \mathbf{F}_{K}} T_{i} T_{j} \omega_{0}\left(p_{\sigma(j)}, p_{\sigma(i)}\right)+\frac{1}{2} \omega_{0}(z(0), z(1)) .
$$

Let $v=z(0) / C$. The above two formulas become, respectively, $\Psi v-v=\sum_{i=1}^{\mathbf{F}_{K}} T_{i} p_{\sigma(i)}$ and

$$
1=\frac{1}{2} \int_{0}^{1}\langle-J \dot{z}, z\rangle d t=\frac{1}{2} C^{2} \sum_{1 \leqslant j<i \leqslant \mathbf{F}_{K}} T_{i} T_{j} \omega_{0}\left(p_{\sigma(j)}, p_{\sigma(i)}\right)+C^{2} \frac{1}{2} \omega_{0}(v, \Psi v) .
$$

By Proposition 3.5 we have $I_{K}(z)=C^{2}$, and thus

$$
I_{K}(z)=\frac{2}{\sum_{1 \leqslant j<i \leqslant \mathbf{F}_{K}} T_{i} T_{j} \omega_{0}\left(p_{\sigma(j)}, p_{\sigma(i)}\right)-\omega_{0}(\Psi v, v)}>0 .
$$

With $E_{\Psi}$ defined as in Theorem 1.1 let

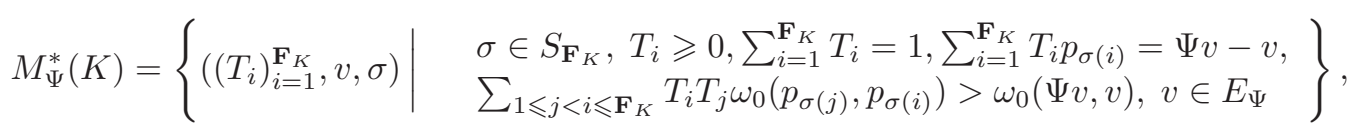

For every triple $\left(\left(T_{i}\right)_{i=1}^{\mathbf{F}_{K}}, v, \sigma\right) \in M_{\Psi}^{*}(K)$, as the construction of $u_{\infty}^{\prime}$ in the proof of Theorem 4.1 we can use it to construct a $z \in \mathcal{A}_{\Psi}^{0}$ such that (4.24) holds. It follows from these and (4.23) that

$$
c_{\mathrm{EHZ}}^{\Psi}(K)=\min _{\left(\left(T_{i}\right)_{i=1}^{\mathbf{F}_{K}}, v, \sigma\right) \in M_{\Psi}^{*}(K)} \frac{2}{\sum_{1 \leqslant j<i \leqslant \mathbf{F}_{K}} T_{i} T_{j} \omega_{0}\left(p_{\sigma(j)}, p_{\sigma(i)}\right)-\omega_{0}(\Psi v, v)},
$$


Let $\beta_{\sigma(i)}=\frac{T_{i}}{h_{\sigma(i)}}$. Since $p_{i}=\frac{2}{h_{i}} J n_{i}$, we get

$$
c_{\mathrm{EHZ}}^{\Psi}(K)=\min _{\left(\left(\beta_{i}\right)_{i=1}^{\left.\mathbf{F}_{K}, v, \sigma\right) \in M_{\Psi}(K)}\right.} \frac{2}{4 \sum_{1 \leqslant j<i \leqslant \mathbf{F}_{K}} \beta_{\sigma(i)} \beta_{\sigma(j)} \omega_{0}\left(n_{\sigma(j)}, n_{\sigma(i)}\right)-\omega_{0}(\Psi v, v)},
$$

where $M_{\Psi}(K)$ is as in Theorem 1.1 .

Step 2. General case. Let $p \in \operatorname{Int}(K)$ be a fixed point of $\Psi$. Consider the symplectomorphism

$$
\phi:\left(\mathbb{R}^{2 n}, \omega_{0}\right) \rightarrow\left(\mathbb{R}^{2 n}, \omega_{0}\right), x \mapsto x-p .
$$

Since $\Psi(p)=p, \phi \circ \Psi=\Psi \circ \phi$ and thus $c_{\mathrm{EHZ}}^{\Psi}(K)=c_{\mathrm{EHZ}}^{\Psi}(\phi(K))$ by the arguments below Proposition 1.2 of $[10]$. Let us write $\hat{K}=\phi(K)$ for convenience. Denote all $(2 n-1)$-dimensional facets of it by $\left\{\hat{F}_{i}\right\}_{i=1}^{\mathbf{F}_{\hat{K}}}$, the unit outer normal to $\hat{F}_{i}$ by $\hat{n}_{i}$, the support function of $\hat{K}$ by $h_{\hat{K}}$. Then $\mathbf{F}_{\hat{K}}=\mathbf{F}_{K}, \hat{F}_{i}=F_{i}-p$ and $\hat{n}_{i}=n_{i}$ for $i=1, \cdots, \mathbf{F}_{K}$, and $h_{\hat{K}}(y)=h_{K}(y)-\langle p, y\rangle$. By Step 1 we get

$$
c_{\mathrm{EHZ}}^{\Psi}(\hat{K})=\min _{\left(\left(\beta_{i}\right)_{i=1}^{\left.\mathbf{F}_{K}, v, \sigma\right) \in M_{\Psi}(\hat{K})}\right.} \frac{2}{4 \sum_{1 \leqslant j<i \leqslant \mathbf{F}_{K}} \beta_{\sigma(i)} \beta_{\sigma(j)} \omega_{0}\left(n_{\sigma(j)}, n_{\sigma(i)}\right)-\omega_{0}(\Psi v, v)},
$$

where with $\hat{h}_{i}=\hat{h}_{\hat{K}}\left(n_{i}\right)=h_{K}\left(n_{i}\right)-\left\langle p, n_{i}\right\rangle=h_{i}-\left\langle p, n_{i}\right\rangle$ for $i=1, \cdots, \mathbf{F}_{K}$,

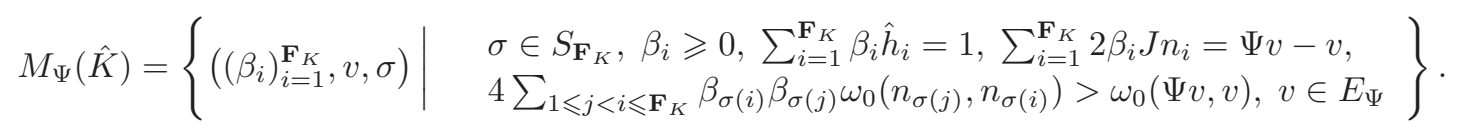

Clearly, it remains to prove $M_{\Psi}(\hat{K})=M_{\Psi}(K)$. In fact, for any $\left(\left(\beta_{i}\right)_{i=1}^{\mathbf{F}_{K}}, v, \sigma\right) \in M_{\Psi}(\hat{K})$, since

$$
1=\sum_{i=1}^{\mathbf{F}_{K}} \beta_{i} \hat{h}_{i}=\sum_{i=1}^{\mathbf{F}_{K}} \beta_{i} h_{i}-\left\langle p, \sum_{i=1}^{\mathbf{F}_{K}} \beta_{i} n_{i}\right\rangle
$$

it suffices to prove $\left\langle p, \sum_{i=1}^{\mathbf{F}_{K}} \beta_{i} n_{i}\right\rangle=0$. Note that $\sum_{i=1}^{\mathbf{F}_{K}} 2 \beta_{i} J n_{i}=\Psi v-v, v \in E_{\Psi}$. We have

$$
\left\langle p, \sum_{i=1}^{\mathbf{F}_{K}} \beta_{i} n_{i}\right\rangle=\omega_{0}\left(p, \sum_{i=1}^{\mathbf{F}_{K}} \beta_{i} J n_{i}\right)=\frac{1}{2} \omega_{0}(p, \Psi v-v)=\frac{1}{2}\left(\omega_{0}(p, \Psi v)-\omega_{0}(p, v)\right)=0
$$

because $\omega_{0}(p, \Psi v)=\omega_{0}(\Psi p, \Psi v)=\omega_{0}(p, v)$. Hence $M_{\Psi}(\hat{K}) \subset M_{\Psi}(K)$, and hence $M_{\Psi}(K) \subset$ $M_{\Psi}(\hat{K})$ since $K=\hat{K}-(-p)$ and $\Psi(-p)=-p$.

\section{Proofs of Theorems $1.2,1.3$}

We have an analogue of Theorem 4.1

Theorem 5.1. Let $K$ be a convex polytope as above (1.3). If $0 \in \operatorname{Int}(K)$, there exists a generalized leafwise chord on $\partial K$ for $\mathbb{R}^{n, k}: \gamma:[0,1] \rightarrow \partial K$ with $A(z)=\min \{A(x) \mid x$ is a generalized leafwise chord on $\partial K$ for $\left.\mathbb{R}^{n, k}\right\}$ such that $\dot{\gamma}$ is piecewise constant and is composed of a finite sequence of vectors, i.e. there exists a sequence of vectors $\left(w_{1}, \ldots, w_{m}\right)$, and a sequence $\left(0=\tau_{0}<\cdots<\tau_{m-1}<\tau_{m}=1\right)$ so that $\dot{\gamma}(t)=w_{i}$ for $\tau_{i-1}<t<\tau_{i}$. Moreover, for each $j \in\{1, \cdots, m\}$ there exists $i \in\left\{1, \cdots, \mathbf{F}_{K}\right\}$ so that $w_{j}=C_{j} J n_{i}$, for some $C_{j}>0$, and for each $i \in\left\{1, \cdots, \mathbf{F}_{K}\right\}$, the set $\left\{t: \exists C>0, \dot{\gamma}(t)=C J n_{i}\right\}$ is connected, i.e. for every $i$ there is at most one $j \in\{1, \ldots, m\}$ with $w_{j}=C_{j} J n_{i}$. Hence there are at most $\mathbf{F}_{K}$ points of discontinuity in $\dot{\gamma}$, and $\gamma$ visits the interior of each facet at most once. 
Proof. Let $z:[0, T] \rightarrow \partial K$ be a generalized leafwise chord with action $A(z)=c_{\mathrm{LR}}(K, K \cap$ $\left.\mathbb{R}^{n, k}\right)=c^{n, k}(K)$ for $\mathbb{R}^{n, k}$. By Theorem 2.2 we can assume it to satisfy (2.14) (by a reparametrization if necessary), and obtain that the path

$$
u:[0,1] \rightarrow \mathbb{R}^{2 n}, t \mapsto \frac{1}{\sqrt{T}} z(T t)-\frac{1}{\sqrt{T}} P_{n, k} \int_{0}^{1} z(T t) d t
$$

belongs to $\mathcal{A}_{2}$ and satisfies $I_{2}(u)=T=c^{n, k}(K)$. Moreover

$$
\dot{u}(t)=\sqrt{T} \dot{z}(T t) \in \sqrt{T} \operatorname{conv}\left\{p_{i} \mid \sqrt{T}(u(t)-b) \in F_{i}\right\} \subset T^{1 / 2} \cdot \operatorname{conv}\left\{p_{1}, \cdots, p_{\mathbf{F}_{K}}\right\}
$$

with $b=-\frac{1}{\sqrt{T}} P_{n, k} \int_{0}^{1} z(T t) d t$ and with $c=T^{1 / 2}$, and so $I_{2}(u)=c^{2}$ by Proposition 3.5

For every $N \in \mathbb{N}$, Lemma 3.1 yields a piecewise affine path $\zeta_{N}$ such that

$$
\left\|u-\zeta_{N}\right\|_{W^{1,2}} \leqslant \frac{1}{N}, \quad \zeta_{N}(0)=u(0), \quad \zeta_{N}(1)=u(1) \quad \text { and } \quad \dot{\zeta}_{N}(t) \in c \cdot \operatorname{conv}\left\{p_{1}, \cdots, p_{\mathbf{F}_{K}}\right\}
$$

for almost every $t$. By applying Lemma 3.3 with $v_{i}=c p_{i}, i=1, \cdots, \mathbf{F}_{K}$ to $\zeta_{N}$, we get a piecewise affine path $\zeta_{N}^{\prime} \in W^{1,2}\left([0,1], \mathbb{R}^{2 n}\right)$ such that

$$
A\left(\zeta_{N}^{\prime}\right) \geq A\left(\zeta_{N}\right), \quad \zeta_{N}^{\prime}(0)=u(0), \quad \zeta_{N}^{\prime}(1)=u(1), \quad \dot{\zeta}_{N}^{\prime}(t) \in\left\{v_{1}, \ldots, v_{\mathbf{F}_{K}}\right\}
$$

for almost every t. Applying Lemma 3.4 to $\zeta_{N}^{\prime}$ again, we can obtain a piecewise affine path $u_{N}:[0,1] \rightarrow \mathbb{R}^{2 n}$ from $u(0)$ to $u(1)$ such that

$$
\dot{u}_{N}(t)=\sum_{i=1}^{m_{N}} \chi_{I_{i}^{N}}(t) v_{i}^{N}
$$

where $v_{i}^{N}=v_{j}$ for some $j \in\left\{1, \cdots, \mathbf{F}_{K}\right\}$ and for every $j$ there is at most one such $i$, and that

$$
A_{N}:=\sqrt{A\left(u_{N}\right)} \geqslant \sqrt{A\left(\zeta_{N}\right)}
$$

Define $u_{N}^{\prime}:=\frac{u_{N}}{A_{N}}$ and $c_{N}=: \frac{c}{A_{N}}$. Notice that $\int_{0}^{1} u_{N}^{\prime}(t) d t$ may not belong to $J V_{0}^{n, k}$ and $u_{N}^{\prime}$ may not belong to $\mathcal{F}_{2}$. Recall that $P_{n, k}: \mathbb{R}^{2 n}=J V_{0}^{n, k} \oplus \mathbb{R}^{n, k} \rightarrow \mathbb{R}^{n, k}$ is the orthogonal projection. Define

$$
y_{N}:=u_{N}^{\prime}-P_{n, k}\left(\int_{0}^{1} u_{N}^{\prime}(t) d t\right)
$$

Then $\int_{0}^{1} y(t) d t \in J V_{0}^{n, k}$ and

$$
\begin{aligned}
A\left(y_{N}\right) & =\int_{0}^{1}\left\langle-J \dot{u}_{N}^{\prime}, u_{N}^{\prime}(t)-P_{n, k}\left(\int_{0}^{1} u_{N}^{\prime}(t) d t\right)\right\rangle d t \\
& =A\left(u_{N}^{\prime}\right)-\left\langle J\left(u_{N}^{\prime}(1)-u_{N}^{\prime}(0)\right), P_{n, k}\left(\int_{0}^{1} u_{N}^{\prime}(t) d t\right)\right\rangle .
\end{aligned}
$$

Since $u_{N}^{\prime}(1)-u_{N}^{\prime}(0) \in V_{0}^{n, k}, A\left(y_{N}\right)=A\left(u_{N}^{\prime}\right)=1$. Thus, $y_{N} \in \mathcal{A}_{2}$. Write $w_{i}^{N}:=\frac{v_{i}^{N}}{A_{N}}$ for the velocities of $y_{N}$, which sits in the set $\frac{c}{A_{N}} \cdot\left\{p_{1}, \cdots, p_{\mathbf{F}_{K}}\right\}$. Since $\left\|u-\zeta_{N}\right\|_{W^{1,2}} \leqslant \frac{1}{N}$ we deduce that $A\left(\zeta_{N}\right) \rightarrow 1$ as $N \rightarrow \infty$. Hence $\underline{\lim }_{N \rightarrow \infty} A_{N} \geqslant 1$, and $\overline{\lim }_{N \rightarrow \infty} c_{N} \leqslant c$. Moreover Proposition 3.5 and the minimality of $I_{2}(u)$ imply that $c_{N}^{2}=I_{K}\left(y_{N}\right) \geqslant I_{K}(u)=c^{2}$. Then $\lim _{N \rightarrow \infty} c_{N}=c$ and thus $\lim _{N \rightarrow \infty} A_{N}=1$.

Recall that the set $\mathcal{A}^{1}$ is defined as above (4.20) and that the map $\Phi$ is as in (4.20). By the proof of Theorem 4.1, the image $\operatorname{Im}(\Phi)$ is contained in the compact subset of $S_{\mathbf{F}_{K}} \times \mathbb{R}^{\mathbf{F}_{K}}$,

$$
S_{\mathbf{F}_{K}} \times\left\{\left(t_{1}, \cdots, t_{\mathbf{F}_{K}}\right) \in \mathbb{R}^{\mathbf{F}_{K}} \mid t_{i} \geqslant 0 \forall i, \sum_{i=1}^{\mathbf{F}_{K}} t_{i}=1\right\} .
$$


Since $y_{N} \in \mathcal{A}^{1}$ with $C=c_{N}$, we can write $\Phi\left(y_{N}\right)=\left(\sigma^{N},\left(t_{1}^{N}, \cdots, t_{\mathbf{F}_{K}}^{N}\right)\right)$. After passing to a subsequence, we can also assume that $\sigma^{N}=\sigma$ is constant, and $\left(t_{1}^{N}, \cdots, t_{\mathbf{F}_{K}}^{N}\right)$ converges to a vector $\left(t_{1}^{\infty}, \cdots, t_{\mathbf{F}_{K}}^{\infty}\right)$. Define

$$
\begin{aligned}
& \tau_{0}^{\infty}=0, \quad \tau_{1}^{\infty}=\tau_{0}^{\infty}+t_{1}^{\infty}, \quad \tau_{j}^{\infty}=\tau_{0}^{\infty}+\sum_{i=1}^{j} t_{i}^{\infty}, \quad j=2, \cdots, \mathbf{F}_{K}, \\
& I_{i}^{\infty}=\left(\tau_{i-1}^{\infty}, \tau_{i}^{\infty}\right), \quad i=1, \cdots, \mathbf{F}_{K}
\end{aligned}
$$

and the piecewise affine path $u_{\infty}^{\prime}(t)=u(0)+\int_{0}^{t} \dot{u}_{\infty}^{\prime}(s) d s$ with

$$
\dot{u}_{\infty}^{\prime}(t)=\sum_{i=1}^{\mathbf{F}_{K}} \chi_{I_{i}^{\infty}}(t) c \cdot p_{\sigma(i)} .
$$

Similar to the proof of Theorem 4.1] one gets $u_{\infty}^{\prime}$ satisfying $u_{\infty}^{\prime}(0)=u(0), u_{\infty}^{\prime}(1)=u(1)$, $A\left(u_{\infty}^{\prime}\right)=1$ and $I_{2}\left(u_{\infty}^{\prime}\right)=c^{2}$. Define

$$
u_{\infty}:=u_{\infty}^{\prime}-P_{n, k}\left(\int_{0}^{1} u_{\infty}^{\prime}(t) d t\right) .
$$

Then $u_{\infty} \in \mathcal{A}_{2}$ and $I_{2}\left(u_{\infty}\right)=T=c^{n, k}(K)$. By Theorem 2.2 we have $\mathbf{a}_{0} \in \mathbb{R}^{n, k}$ such that

$$
[0,1] \ni t \mapsto \gamma(t):=\sqrt{T} u_{\infty}(t)+\mathbf{a}_{0} / \sqrt{T}
$$

is a piecewise affine generalized leafwise chord on $\partial K$ for $\mathbb{R}^{n, k}$ with action

$$
A(\gamma)=I_{2}(u)=c_{\mathrm{LR}}\left(K, K \cap \mathbb{R}^{n, k}\right)
$$

and satisfying $\dot{\gamma}(t) \in T \cdot\left\{p_{1}, \cdots, p_{\mathbf{F}_{K}}\right\}$ for almost every $t \in[0,1]$ and that the set $\left\{t: \dot{\gamma}(t)=p_{i}\right\}$ is connected for every $i$. Recall $p_{i}=\frac{2}{h_{i}} J n_{i}$. Theorem 5.1 is proved.

Proof of Theorem 1.2. Step 1. Case $0 \in \operatorname{Int}(K)$. Let $\mathcal{A}_{2}^{0}$ consist of $z \in \mathcal{A}_{2}$ for which there exist $C>0$ and an increasing sequence of numbers $0=\tau_{0} \leq \tau_{1} \leq \ldots \leq \tau_{\mathbf{F}_{K}}=1$ such that

$$
\dot{z}(t)=\sum_{i=1}^{\mathbf{F}_{K}} \chi_{I_{i}}(t) C \cdot p_{\sigma(i)}
$$

with $I_{i}=\left(\tau_{i-1}, \tau_{i}\right)$, where $\sigma \in S_{\mathbf{F}_{K}}$ is the permutation on $\left\{1, \cdots, \mathbf{F}_{K}\right\}$. Then $u_{\infty}^{\prime}$ in the proof of Theorem 5.1 belongs to $\mathcal{A}_{2}^{0}$ and satisfies $I_{K}\left(u_{\infty}^{\prime}\right)=c_{\mathrm{LR}}\left(K, K \cap \mathbb{R}^{n, k}\right)$. Thus

$$
c_{\mathrm{LR}}\left(K, K \cap \mathbb{R}^{n, k}\right)=\min \left\{I_{2}(z) \mid z \in \mathcal{A}_{2}\right\}=\min \left\{I_{2}(z) \mid z \in \mathcal{A}_{2}^{0}\right\} .
$$

For any $z \in \mathcal{A}_{2}^{0}$, we have $z(0), z(1) \in \mathbb{R}^{n, k}, \dot{z}$ has the form of (5.26) and hence

$$
V_{0}^{n, k} \ni z(1)-z(0)=\int_{0}^{1} \dot{z}(t) d t=C \sum_{i=1}^{\mathbf{F}_{K}} T_{i} p_{\sigma(i)}
$$

where $T_{i}=\left|I_{i}\right|$, and Proposition 3.2 yields

$$
1=\frac{1}{2} \int_{0}^{1}\langle-J \dot{z}, z\rangle d t=\frac{1}{2} C^{2} \sum_{1 \leqslant j<i \leqslant \mathbf{F}_{K}} T_{i} T_{j} \omega_{0}\left(p_{\sigma(j)}, p_{\sigma(i)}\right)+\frac{1}{2} \omega_{0}(z(0), z(1)) .
$$

Note that $\omega_{0}(z(0), z(1))=\omega_{0}(z(0), z(1)-z(0))=0$, and $I_{2}(z)=C^{2}$ by Proposition 3.5 . Then

$$
I_{2}(z)=\frac{2}{\sum_{1 \leqslant j<i \leqslant \mathbf{F}_{K}} T_{i} T_{j} \omega_{0}\left(p_{\sigma(j)}, p_{\sigma(i)}\right)}>0 .
$$


Let

$$
M^{*}(K)=\left\{\begin{array}{l|l}
\left(\left(T_{i}\right)_{i=1}^{\mathbf{F}_{K}}, \sigma\right) \mid \begin{array}{l}
\sigma \in S_{\mathbf{F}_{K}}, T_{i} \geqslant 0, \sum_{i=1}^{\mathbf{F}_{K}} T_{i}=1, \sum_{i=1}^{\mathbf{F}_{K}} T_{i} p_{\sigma(i)} \in V_{0}^{n, k} \\
\sum_{1 \leqslant j<i \leqslant \mathbf{F}_{K}} T_{i} T_{j} \omega_{0}\left(p_{\sigma(j)}, p_{\sigma(i)}\right)>0
\end{array}
\end{array}\right\} .
$$

For every pair $\left(\left(T_{i}\right)_{i=1}^{\mathbf{F}_{K}}, \sigma\right) \in M^{*}(K)$, as in the construction of $u_{\infty}^{\prime}$ in the proof of Theorem 4.1 we can use $\left(\left(T_{i}\right)_{i=1}^{\mathbf{F}_{K}}, \sigma\right)$ to construct a $z \in \mathcal{A}_{2}^{0}$ such that (5.28) holds. It follows that

$$
c_{\mathrm{LR}}\left(K, K \cap \mathbb{R}^{n, k}\right)=\min _{\left(\left(T_{i}\right)_{i=1}^{\left.\mathbf{F}_{K}, \sigma\right) \in M^{*}(K)}\right.} \frac{2}{\sum_{1 \leqslant j<i \leqslant \mathbf{F}_{K}} T_{i} T_{j} \omega_{0}\left(p_{\sigma(j)}, p_{\sigma(i)}\right)},
$$

Define $\beta_{\sigma(i)}:=\frac{T_{i}}{h_{\sigma(i)}}$. Since $p_{i}=\frac{2}{h_{i}} J n_{i}$, The above two formulas give the desired formula in this case.

Step 2. General case. Let $p \in \operatorname{Int}(K) \cap \mathbb{R}^{n, k}$. Then the symplectomorphism $\phi$ defined by (4.25) satisfies $c_{\mathrm{LR}}\left(\phi(K), \phi(K) \cap \mathbb{R}^{n, k}\right)=c_{\mathrm{LR}}\left(K, K \cap \mathbb{R}^{n, k}\right)$ by the arguments at the beginning of [11, §3]. As in Step 2 of the proof of Theorem 1.1 let $\hat{K}=\phi(K)$. By Step 1 we obtain

$$
c_{\mathrm{LR}}\left(\hat{K}, \hat{K} \cap \mathbb{R}^{n, k}\right)=\frac{1}{2} \min _{\left(\left(\beta_{i}\right)_{i=1}^{\left.\mathbf{F}_{K}, \sigma\right) \in M(\hat{K})}\right.} \frac{1}{\sum_{1 \leqslant j<i \leqslant \mathbf{F}_{K}} \beta_{\sigma(i)} \beta_{\sigma(j)} \omega_{0}\left(n_{\sigma(j)}, n_{\sigma(i)}\right)},
$$

where

$$
M(\hat{K})=\left\{\left(\left(\beta_{i}\right)_{i=1}^{\mathbf{F}_{K}}, \sigma\right) \mid \begin{array}{l}
\beta_{i} \geqslant 0, \sum_{i=1}^{\mathbf{F}_{K}} \beta_{i} \hat{h}_{i}=1, \sum_{i=1}^{\mathbf{F}_{K}} \beta_{i} J n_{i} \in V_{0}^{n, k}, \\
\sum_{1 \leqslant j<i \leqslant \mathbf{F}_{K}} \beta_{\sigma(i)} \beta_{\sigma(j)} \omega_{0}\left(n_{\sigma(j)}, n_{\sigma(i)}\right)>0, \sigma \in S_{\mathbf{F}_{K}}
\end{array}\right\} .
$$

Now we are in position to prove that $M(\hat{K})$ is equal to $M(K)$ in (1.6). We only need to prove $M(\hat{K}) \subset M(K)$ because of obvious reasons. Since $\left(\left(\beta_{i}\right)_{i=1}^{\mathbf{F}_{K}}, \sigma\right) \in M(\hat{K})$ satisfies

$$
1=\sum_{i=1}^{\mathbf{F}_{K}} \beta_{i} \hat{h}_{i}=\sum_{i=1}^{\mathbf{F}_{K}} \beta_{i} h_{i}-\left\langle p, \sum_{i=1}^{\mathbf{F}_{K}} \beta_{i} n_{i}\right\rangle,
$$

it suffices to prove $\left\langle p, \sum_{i=1}^{\mathbf{F}_{K}} \beta_{i} n_{i}\right\rangle=0$. Note that $\sum_{i=1}^{\mathbf{F}_{K}} \beta_{i} J n_{i} \in V_{0}^{n, k}$. We have

$$
\left\langle p, \sum_{i=1}^{\mathbf{F}_{K}} \beta_{i} n_{i}\right\rangle=\omega_{0}\left(p, \sum_{i=1}^{\mathbf{F}_{K}} \beta_{i} J n_{i}\right)=0
$$

because $\mathbb{R}^{n, k}$ and $V_{0}^{n, k}$ are $\omega_{0}$-orthogonal. Hence $M(\hat{K}) \subset M(K)$.

Proof of Theorem 1.3. Let $p \in D \cap L \cap \mathbb{R}^{1,0}$, define $\phi: \mathbb{R}^{2} \rightarrow \mathbb{R}^{2}, x \mapsto x-p$. As in [11, §3] we have $c_{\mathrm{LR}}\left(D, D \cap \mathbb{R}^{1,0}\right)=c_{\mathrm{LR}}\left(\phi(D), \phi(D) \cap \mathbb{R}^{1,0}\right)$ and

$c_{\mathrm{LR}}\left(D_{1}, D_{1} \cap \mathbb{R}^{1,0}\right)=c_{\mathrm{LR}}\left(\phi\left(D_{1}\right), \phi\left(D_{1}\right) \cap \mathbb{R}^{1,0}\right), \quad c_{\mathrm{LR}}\left(D_{2}, D_{2} \cap \mathbb{R}^{1,0}\right)=c_{\mathrm{LR}}\left(\phi\left(D_{2}\right), \phi\left(D_{2}\right) \cap \mathbb{R}^{1,0}\right)$.

Thus we can assume $0 \in D \cap L \cap \mathbb{R}^{1,0}$ below.

Let $H^{+}:=\left\{(x, y) \in \mathbb{R}^{2} \mid y \geqslant 0\right\}, H^{-}:=\left\{(x, y) \in \mathbb{R}^{2} \mid y \leqslant 0\right\}$, and write $K^{+}=H^{+} \cap K$ and $K^{-}=H^{-} \cap K$ for any subset $K \subset \mathbb{R}^{2}$. On each of $\partial D, \partial D_{1}$ and $\partial D_{2}$ there only exist two generalized leafwise chords for $\mathbb{R}^{1,0}$, that is, $(\partial D)^{+}$and $(\partial D)^{-}$on $\partial D,\left(\partial D_{1}\right)^{+}$and $\left(\partial D_{1}\right)^{-}$on $\partial D_{1},\left(\partial D_{2}\right)^{+}$and $\left(\partial D_{2}\right)^{-}$on $\partial D_{2}$. Note that a GLC $x$ on $\partial D$ for $\mathbb{R}^{1,0}$ and the line segment $D \cap \mathbb{R}^{1,0}$ form a loop $\gamma$ and that $\langle-J \dot{z}, z\rangle$ vanishes along the line segment $D \cap \mathbb{R}^{1,0}$. Using these and Stokes theorem we deduce that $A(x)=\int_{x} q d p=\int_{\gamma} q d p$ is equal to the symplectic area of the domain surrounded by $\gamma$. Hence

$$
\begin{aligned}
& c_{\mathrm{LR}}\left(D, D \cap \mathbb{R}^{1,0}\right)=\min \left\{\operatorname{Area}\left(D^{+}\right), \operatorname{Area}\left(D^{-}\right)\right\}, \\
& c_{\mathrm{LR}}\left(D_{1}, D_{1} \cap \mathbb{R}^{1,0}\right)=\min \left\{\operatorname{Area}\left(D_{1}^{+}\right), \operatorname{Area}\left(D_{1}^{-}\right)\right\}, \\
& c_{\mathrm{LR}}\left(D_{2}, D_{2} \cap \mathbb{R}^{1,0}\right)=\min \left\{\operatorname{Area}\left(D_{2}^{+}\right), \operatorname{Area}\left(D_{2}^{-}\right)\right\} .
\end{aligned}
$$


Assume without loss of generality that $c_{\mathrm{LR}}\left(D, D \cap \mathbb{R}^{1,0}\right)=\operatorname{Area}\left(D^{+}\right)$. Then

$$
\begin{aligned}
c_{\mathrm{LR}}\left(D_{1}, D_{1} \cap \mathbb{R}^{1,0}\right)+c_{\mathrm{LR}}\left(D_{2}, D_{2} \cap \mathbb{R}^{1,0}\right) & \leqslant \operatorname{Area}\left(D_{1} \cap D^{+}\right)+\operatorname{Area}\left(D_{2} \cap D^{+}\right) \\
& =\operatorname{Area}\left(D^{+}\right)=c_{\mathrm{LR}}\left(D, D \cap \mathbb{R}^{1,0}\right) .
\end{aligned}
$$

\section{References}

[1] A. Artstein-Avidan and Y. Ostrover, Bounds for Minkowski billiard trajectories in convex bodies, Int. Math. Res. Not., (2012),165-193.

[2] A. Akopyan, R. Karasev, and F. Petrov, Bang's problem and symplectic invariants, J. Symplectic Geom., 17(2019), no. 6, 1579-1611.

[3] F. H. Clarke, On Hamiltonian flows and symplectic transformations, SIAM J. Control Optim., 20(1982), no. 3, 355-359.

[4] F. H. Clarke, Optimization and nonsmooth analysis. Canadian Mathematical Society Series of Monographs and Advanced Texts. A Wiley-Interscience Publication. John Wiley \& Sons, Inc., New York, 1983.

[5] I. Ekeland, Convexity methods in Hamiltonian mechanics, Ergebnisse der Mathematik und ihrer Grenzgebiete (3) [Results in Mathematics and Related Areas (3)], 19. SpringerVerlag, Berlin, 1990.

[6] I. Ekeland and H. Hofer, Symplectic topology and Hamiltonian dynamics, Math. Z., 200(1989), 355-378.

[7] I. Ekeland, Hamilton-Jacobi on the symplectic group, Rend. Istit. Mat. Univ. Trieste, 49(2017), 137-146.

[8] H. Hofer and E. Zehnder, A new capacity for symplectic manifolds, Analysis et cetera 1990, 405-429.

[9] Rongrong Jin, Guangcun Lu, Representation formula for symmetrical symplectic capacity and applications, Discrete Contin. Dyn. Syst., 40(2020), no. 8, 4705-4765.

[10] Rongrong Jin, Guangcun Lu, Generalizations of Ekeland-Hofer and Hofer-Zehnder symplectic capacities and applications, arXiv:1903.01116v2[math.SG] 16 May 2019.

[11] Rongrong Jin, Guangcun Lu, Representation formula for coisotropic Hofer-Zehnder capacity of convex bodies and related results, arXiv:1909.08967v1[math.SG] 19 September 2019.

[12] Rongrong Jin, Guangcun Lu, Coisotropic Ekeland-Hofer capacities, arXiv:1910.14474.

[13] S. Lisi, A. Rieser, Coisotropic Hofer-Zehnder capacities and non-squeezing for relative embeddings, J. Symplectic Geom., 18(2020), no. 3, 819-865.

[14] C. G. Liu and Q. Wang, Symmetrical symplectic capacity with applications, Discrete Contin. Dyn. Syst., 32(2012), 2253-2270. (MR2885809) [10.3934/dcds.2012.32.2253]

[15] Pazit Haim-Kislev, On the symplectic size of convex polytopes, Geometric And Functional Analysis, 29(2019), 440-463.

[16] J.-C. Sikorav, Systémes Hamiltoniens et topologie symplectique. Dipartimento di Matematica dell'Universitá di Pisa, 1990. ETS, EDITRICE PISA.

School of Mathematical Sciences, Beijing Normal University

Laboratory of Mathematics and Complex Systems, Ministry of Education

Beijing 100875, The People's Republic of China

E-mail address: shikun@mail.bnu.edu.cn, gclu@bnu.edu.cn 